\title{
FAILURE IDENTIFICATION FOR 3D LINEAR SYSTEMS
}

\author{
S. Maleki • P. Rapisarda • L. \\ Ntogramatzidis • E. Rogers
}

the date of receipt and acceptance should be inserted later

\begin{abstract}
Geometric control theory is used to investigate the problem of fault detection and isolation for 3D linear systems described by FornasiniMarchesini models with the aim using these results in applications areas such as wireless sensor networks.. Necessary and sufficient conditions for the existence of a solution to this problem are established together with constructive methods for the design of observers for fault detection and identification.
\end{abstract}

Keywords 3D systems · fault detection · fault identification · FornasiniMarchesini model

\section{Introduction}

Fault detection and isolation is a well developed area in the standard, or $1 \mathrm{D}$, systems setting with a large supporting literature including the survey papers $[8,9]$. Comparatively little literature is available for the same problem in the $n \mathrm{D}$ setting is much less rich. Moreover, previous research is, in the main, for $2 \mathrm{D}$ systems, and based on polynomial-algebraic tools, see $[2,5,19]$.

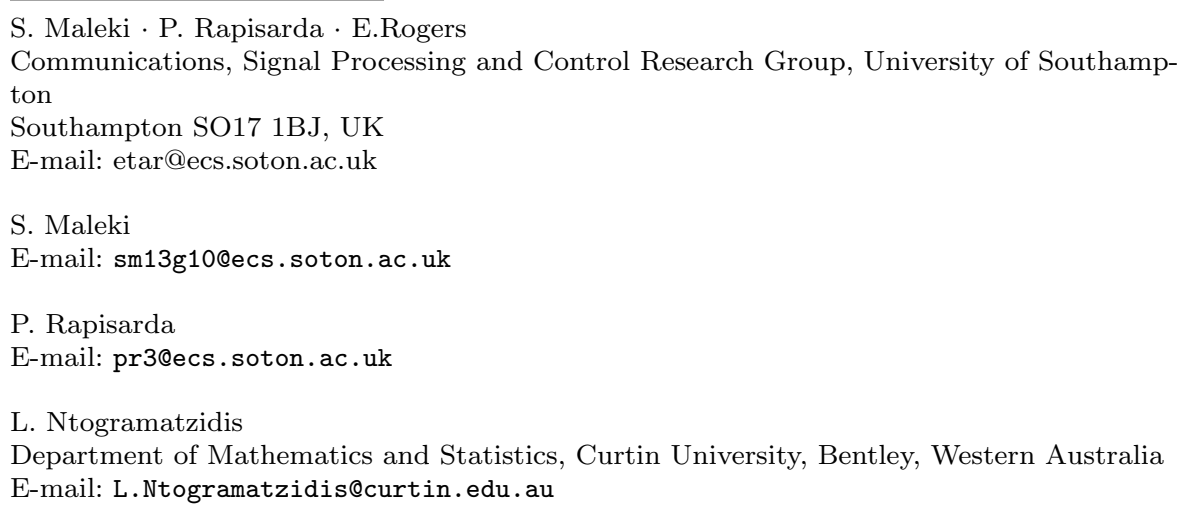


This paper uses the more recently developed geometric framework for $2 \mathrm{D}$ systems (see [14-16]) to investigate the problem of fault detection and isolation for 3D Fornasini-Marchesini models. Motivation for this work comes from the use of these models to represent the dynamics of wireless sensor networks, see [3], where such networks are currently the subject of a high level of research effort. Such networks are intrinsically 3D systems and for this reason attention is restricted to this case, with routine extension of the results obtained to $n \mathrm{D}$, $n>3$ systems. The geometric approach to $3 \mathrm{D}$ fault detection and isolation was previously addressed in [12], where 1D geometric theory, see [1,18], was applied to several different formulations of the fault detection and isolation problem.

In common with [12], the general problem considered is how to define functions, termed residuals in the remainder of this paper, of the three independent variables such that they are close to zero when no fault is present and their directional properties give information on the presence and nature of a fault when a failure has occurred. Necessary and sufficient conditions for the residuals to provide sufficient information to solve the fault detection and isolation problem are developed together with constructive methods for the design of the observers associated with the residuals.

The motivation for this work is to apply multidimensional systems theory to solve problems relating to the implementation and reliability of wireless sensor networks for applications in the agricultural and environmental monitoring sectors and, in particular, contaminant propagation detection and structural health monitoring. In these and other application areas, grid or mesh topologies are a preferred option and this has, in turn, led to the grid sensor approach. To be of use, such networks must have high reliability and using distributed schemes for signal/information processing in these networks has immediate and significant benefits in terms energy consumption and data throughput. Distributed algorithms also support applications where local actuation is required in response to local detection and hence minimum response delays when compared with a centralized approach. Implementation of a grid sensor network can be achieved with a 3D Fornasini Marchesini state-space model [3], with two spatial and a time indeterminate. With this motivation, the results in this paper considers the $3 \mathrm{D}$ case but there is a natural generalization to $n \mathrm{D}$ systems, $n>3$.

The paper is organized as follows: in sections 2 and 3 we generalize the results of the $2 \mathrm{D}$ geometric approach to the $3 \mathrm{D}$ situation. In most cases the concepts and notions generalize in a straightforward way, but with the specific wireless sensor networks application area in mind, the input-containing conditioned invariant subspaces are considered in detail (section 3.2) and this leads to the introduction of the new concept of the unobservability subspace (section 3.3). In section 4 we consider the computation of externally- and internally stabilizing gains for the 3D case. In section 5 we show how sensor-and actuator failures can be modeled as disturbances on 3D Fornasini-Marchesini models. The statement of the failure detection and identification problem considered in this paper is given in section 6 , and in sections 6.1 and 6.2 we give 
necessary and sufficient conditions for its solution, and constructive methods for the computation of residual generators. Section 7 discusses the limitations of our approach, and some directions for future research.

\section{Notation}

We denote by $\mathbb{R}^{m \times n}$ the set of all $m \times n$ matrices with entries in $\mathbb{R}$. $\mathbb{R}^{\bullet \times n}$ denotes the set of matrices with $n$ columns and an unspecified (but finite) number of rows. If $A_{i} \in \mathbb{R}^{m \times n}, i=1,2,3$, we denote by $A_{H}$ the matrix

$$
A_{H}:=\left[\begin{array}{lll}
A_{1} & A_{2} & A_{3}
\end{array}\right] \in \mathbb{R}^{m \times 3 n},
$$

and by $A_{D}$ the matrix

$$
A_{D}:=\left[\begin{array}{ccc}
A_{1} & 0_{m \times n} & 0_{m \times n} \\
0_{m \times n} & A_{2} & 0_{m \times n} \\
0_{m \times n} & 0_{m \times n} & A_{3}
\end{array}\right] \in \mathbb{R}^{3 m \times 3 n} .
$$

Given $A \in \mathbb{R}^{m \times n}$, we denote by $A^{\dagger}$ its Moore-Penrose pseudo-inverse.

$\mathbb{W}^{\mathbb{T}}$ denotes the set consisting of all trajectories from $\mathbb{T}$ to $\mathbb{W} . \sigma_{i}, i=1,2,3$ are the forward shift operators $\sigma_{i}:\left(\mathbb{R}^{\mathrm{w}}\right)^{\mathbb{Z} \times \mathbb{Z} \times \mathbb{Z}} \rightarrow\left(\mathbb{R}^{\mathrm{w}}\right)^{\mathbb{Z} \times \mathbb{Z} \times \mathbb{Z}}, i=1,2,3$ defined by

$$
\begin{aligned}
& \left(\sigma_{1} w\right)\left(k_{1}, k_{2}, k_{3}\right):=w\left(k_{1}+1, k_{2}, k_{3}\right) \\
& \left(\sigma_{2} w\right)\left(k_{1}, k_{2}, k_{3}\right):=w\left(k_{1}, k_{2}+1, k_{3}\right) \\
& \left(\sigma_{3} w\right)\left(k_{1}, k_{2}, k_{3}\right):=w\left(k_{1}, k_{2}, k_{3}+1\right) .
\end{aligned}
$$

The composition of $i$ times the first shift, $j$ times the second, and $k$ times the third is denoted by $\sigma_{1}^{i} \sigma_{2}^{j} \sigma_{3}^{k}$.

Given a subspace $\mathcal{V} \subseteq \mathbb{R}^{n}$, the notation $\mathcal{V} \dot{\oplus} \mathcal{V} \dot{\mathcal{V}}$ denotes the subspace of $\mathbb{R}^{3 n}$ defined by

$$
\mathcal{V} \dot{\oplus} \mathcal{V} \dot{\oplus} \mathcal{V}:=\left\{\left[\begin{array}{l}
v_{1} \\
v_{2} \\
v_{3}
\end{array}\right] \mid v_{i} \in \mathcal{V}, i=1,2,3\right\}
$$

and $\mathcal{V} \dot{\oplus} \mathcal{V} \dot{\oplus} \mathcal{V} \dot{\oplus} \mathbb{R}^{m}$ denotes the subspace of $\mathbb{R}^{3 n+m}$ defined by

$$
\mathcal{V} \dot{\oplus} \mathcal{V} \dot{\oplus} \mathcal{V} \dot{\oplus} \mathbb{R}^{m}:=\left\{\left[\begin{array}{c}
v_{1} \\
v_{2} \\
v_{3} \\
x
\end{array}\right] \mid v_{i} \in \mathcal{V}, i=1,2,3, \text { and } x \in \mathbb{R}^{m}\right\}
$$

Finally, if $x \in \mathbb{R}^{n},\|x\|$ denotes its Euclidean norm. 


\section{Invariant subspaces}

The purpose of this section is to show how the basic definitions and concepts introduced in [14-16] can be generalized to the 3D case.

In this paper we consider the following class of Fornasini-Marchesini (FM) models:

$$
\begin{aligned}
\sigma_{1} \sigma_{2} \sigma_{3} x= & A_{1} \sigma_{2} \sigma_{3} x+A_{2} \sigma_{1} \sigma_{3} x+A_{3} \sigma_{1} \sigma_{2} x \\
& +B_{1} \sigma_{2} \sigma_{3} u+B_{2} \sigma_{1} \sigma_{3} u+B_{3} \sigma_{1} \sigma_{2} u \\
y= & C x
\end{aligned}
$$

where $A_{i} \in \mathbb{R}^{n \times n}, B_{i} \in \mathbb{R}^{n \times m}, i=1,2,3, C \in \mathbb{R}^{p \times n}, x(i, j, k) \in \mathbb{R}^{n}$ is the local state vector, $y(i, j, k) \in \mathbb{R}^{q}$ is the output vector and $u(i, j, k) \in \mathbb{R}^{m}$ is the input vector.

Definition 1 A subspace $\mathcal{V} \subseteq \mathbb{R}^{n}$ is $\left(A_{1}, A_{2}, A_{3}\right)$-invariant if $A_{i} \mathcal{V} \subseteq \mathcal{V}, i \in$ $\{1,2,3\}$.

The following result gives several characterizations of $\left(A_{1}, A_{2}, A_{3}\right)$-invariance.

Proposition 1 Let $\mathcal{V} \subseteq \mathbb{R}^{n}$ be a subspace of dimension $r$, and let $Q \in$ $\mathbb{R}^{(n-r) \times n}$ and $V \in \mathbb{R}^{n \times r}$ be respectively full row-rank and full column-rank matrices such that $\operatorname{im}(V)=\operatorname{ker}(Q)=\mathcal{V}$.

The following statements are equivalent:

I. $\mathcal{V}$ is $\left(A_{1}, A_{2}, A_{3}\right)$-invariant;

II. There exist matrices $X_{i} \in \mathbb{R}^{r \times r}, i=1,2,3$, such that $A_{i} V=V X_{i}$, or equivalently

$$
\left[\begin{array}{l}
A_{1} \\
A_{2} \\
A_{3}
\end{array}\right] V=\left[\begin{array}{ccc}
V & 0_{n \times r} & 0_{n \times r} \\
0_{n \times r} & V & 0_{n \times r} \\
0_{n \times r} & 0_{n \times r} & V
\end{array}\right]\left[\begin{array}{c}
X_{1} \\
X_{2} \\
X_{3}
\end{array}\right]
$$

III. $A_{H}(\mathcal{V} \dot{\oplus} \mathcal{V} \dot{\oplus} \mathcal{V}) \subseteq \mathcal{V}$.

$I V$. There exist $L_{i} \in \mathbb{R}^{(n-r) \times(n-r)}$ such that $Q A_{i}=L_{i} Q, i=1,2,3$.

Proof Follows from arguments similar to those used in section 2.1 of [16], and is thus omitted.

Given statement $I I I$ of Prop. 1 , we sometimes refer to $A_{H}$-invariance rather than $\left(A_{1}, A_{2}, A_{3}\right)$-invariance. We state separately for ease of future use the following further characterization of $A_{H}$-invariance.

Theorem 1 Let $\mathcal{V} \subseteq \mathbb{R}^{n}$ be a subspace of dimension $r$. The following statements are equivalent:

I. $\mathcal{V}$ is $\left(A_{1}, A_{2}, A_{3}\right)$-invariant;

II. There exists a similarity transformation $T \in \mathbb{R}^{n \times n}$ such that

$$
\hat{A}_{i} \triangleq T^{-1} A_{i} T=\left[\begin{array}{cc}
\hat{A}_{i}^{11} & \hat{A}_{i}^{12} \\
0_{(n-r) \times r} & \hat{A}_{i}^{22}
\end{array}\right], i=1,2,3 .
$$


Proof Follows from arguments similar to those used to prove Theorem 2.1 of [14].

It follows from Theorem 1 that if $\mathcal{V}$ is $A_{H}$-invariant then, without loss of generality, it can be assumed that the FM model (1) is of the form

$$
\begin{aligned}
{\left[\begin{array}{c}
x_{i+1, j+1, k+1}^{\prime} \\
x_{i+1, j+1, k+1}^{\prime \prime}
\end{array}\right]=} & {\left[\begin{array}{cc}
\hat{A}_{1}^{11} & \hat{A}_{1}^{12} \\
0_{(n-r) \times r} & \hat{A}_{1}^{22}
\end{array}\right]\left[\begin{array}{l}
x_{i, j+1, k+1}^{\prime} \\
x_{i, j+1, k+1}^{\prime \prime}
\end{array}\right]+\left[\begin{array}{cc}
\hat{A}_{2}^{11} & \hat{A}_{2}^{12} \\
0_{(n-r) \times r} & \hat{A}_{2}^{22}
\end{array}\right]\left[\begin{array}{l}
x_{i+1, j, k+1}^{\prime} \\
x_{i+1, j, k+1}^{\prime \prime}
\end{array}\right] } \\
+ & {\left[\begin{array}{cc}
\hat{A}_{3}^{11} & \hat{A}_{3}^{12} \\
0_{(n-r) \times r} & \hat{A}_{3}^{22}
\end{array}\right]\left[\begin{array}{l}
x_{i+1, j+1, k}^{\prime} \\
x_{i+1, j+1, k}^{\prime \prime}
\end{array}\right] } \\
& +B_{1}^{\prime} u_{i, j+1, k+1}+B_{2}^{\prime} u_{i+1, j, k+1}+B_{3}^{\prime} u_{i+1, j+1, k} \\
y_{i, j, k}= & {\left[C^{\prime} C^{\prime \prime}\right]\left[\begin{array}{l}
x_{i, j, k}^{\prime} \\
x_{i, j, k}^{\prime \prime}
\end{array}\right]+D u_{i, j, k} . }
\end{aligned}
$$

We use the representation (4) in order to introduce and study the concept of internal and external stability, first introduced in the 2D case in [14]. For $L \in \mathbb{Z}$ we define

$$
\mathcal{S}_{L}:=\left\{(i, j, k) \in \mathbb{Z}^{3} \mid i+j+k=L\right\} .
$$

If $\mathcal{V}$ is an $\left(A_{1}, A_{2}, A_{3}\right)$-invariant subspace and we assign boundary conditions $\left\{x_{i, j, k} \mid(i, j, k) \in \mathcal{S}_{0}\right\} \subset \mathcal{V}$ and $u_{i, j, k}:=0$ for all $(i, j, k) \in \mathbb{Z}^{3}$, then the sequence $x$ compatible with the equations (4) satisfies $x_{i, j, k} \in \mathcal{V}$ for all $(i, j, k) \in \mathbb{Z}^{3}$, i.e.

$$
x_{i, j, k}=\left[\begin{array}{c}
x_{i, j, k}^{\prime} \\
0_{(n-r) \times 1}
\end{array}\right] .
$$

Definition 2 An $\left(A_{1}, A_{2}, A_{3}\right)$-invariant subspace $\mathcal{V}$ is internally stable if

$$
\begin{aligned}
& {\left[\left\{x_{i, j, k} \mid(i, j, k) \in \mathcal{S}_{0}\right\} \subset \mathcal{V}\right] \text { and }\left[u_{i, j, k}:=0 \text { for all }(i, j, k) \in \mathbb{Z}^{3}\right]} \\
& \Longrightarrow \lim _{i, j, k \rightarrow \infty}\left\|x_{i, j, k}^{\prime}\right\|=0 .
\end{aligned}
$$

It follows from standard results in $n \mathrm{D}$ systems theory (see for example [10]) that $\mathcal{V}$ is internally stable if and only if the matrices $A_{i}^{11}, i=1,2,3$ satisfy

$$
\begin{aligned}
& \operatorname{det}\left(I_{n}-A_{1}^{11} \lambda-A_{2}^{11} \mu-A_{3}^{11} \nu\right) \neq 0 \\
& \text { for all }(\lambda, \mu, \nu) \in\left\{\left(z_{1}, z_{2}, z_{3}\right) \in \mathbb{C}^{3}|| z_{i} \mid \leq 1, i=1,2,3\right\} .
\end{aligned}
$$

Definition 3 An $\left(A_{1}, A_{2}, A_{3}\right)$-invariant subspace $\mathcal{V}$ is externally stable if for all

$$
\begin{aligned}
& {\left[\left\{x_{i, j, k} \mid(i, j, k) \in \mathcal{S}_{0}\right\} \nsubseteq \mathcal{V}\right] \text { and }\left[u_{i, j, k}:=0,(i, j, k) \in \mathbb{Z}^{3}\right]} \\
& \Longrightarrow \lim _{i, j, k \rightarrow \infty} x_{i, j, k}^{\prime \prime} \in \mathcal{V} .
\end{aligned}
$$


It is straightforward to check that $\mathcal{V}$ is externally stable if and only if the triple $\left(A_{1}^{22}, A_{2}^{22}, A_{3}^{22}\right)$ is asymptotically stable in the sense of (6).

The condition (6) is rather difficult to check, and is not easy to use in the synthesis of stabilizing controllers. These issues have led to the use of LMIs, see for example $[7,11]$, for this purpose. The following result is a restatement for the 3D case of the main result in [11].

Proposition 2 If there exist $P_{i}=P_{i}^{\top} \in \mathbb{R}^{n}, P_{i}>0, i=1,2,3$, such that the following LMI holds:

$$
\left[\begin{array}{ccc}
P_{1} & 0 & 0 \\
0 & P_{2} & 0 \\
0 & 0 & P_{3}
\end{array}\right]-\left[\begin{array}{l}
A_{1}^{\top} \\
A_{2}^{\top} \\
A_{3}^{\top}
\end{array}\right]\left(P_{1}+P_{2}+P_{3}\right)\left[A_{1} A_{2} A_{3}\right]>0,
$$

then the $3 D$ system described by

$$
x_{i+1, j+1, k+1}=A_{1} x_{i, j+1, k+1}+A_{2} x_{i+1, j, k+1}+A_{3} x_{i+1, j+1, k}
$$

is asymptotically stable.

Proof The proof is analogous to that of Theorem 1 in [11] and is omitted.

\section{Conditioned invariant subspaces}

3.1 Definition and characterizations

Definition 4 A subspace $\mathcal{V} \subseteq \mathbb{R}^{n}$ is $\left(A_{H}, C_{D}\right)$-conditioned invariant for (1), if

$$
\left[\begin{array}{lll}
A_{1} & A_{2} & A_{3}
\end{array}\right]\left(\mathcal{V} \dot{\oplus} \mathcal{V} \dot{\oplus} \mathcal{V} \bigcap \text { ker }\left[\begin{array}{lll}
C & 0 & 0 \\
0 & C & 0 \\
0 & 0 & C
\end{array}\right]\right) \subseteq \mathcal{V} .
$$

The set of conditioned invariants is closed under intersection.

We give a number of characterizations of $\left(A_{H}, C_{D}\right)$-invariance.

Proposition 3 Let $\mathcal{V}$ be a $r$-dimensional subspace of $\mathbb{R}^{n}$, and let $Q \in \mathbb{R}^{(n-r) \times n}$ be a full rank matrix such that $\operatorname{ker}(Q)=\mathcal{V}$. The following statements are equivalent:

I. $\mathcal{V}$ is $\left(A_{H}, C_{D}\right)$-conditioned invariant;

II. There exist $\Gamma=\left[\begin{array}{lll}\Gamma_{1} & \Gamma_{2} & \Gamma_{3}\end{array}\right] \in \mathbb{R}^{(n-r) \times 3(n-r)}$ and $\Lambda \in\left[\begin{array}{lll}\Lambda_{1} & \Lambda_{2} & \Lambda_{3}\end{array}\right] \in$ $\mathbb{R}^{(n-r) \times 3 p}$ such that

$$
Q A_{H}=\Gamma Q_{D}+\Lambda C_{D},
$$

or equivalently there exist $\Gamma_{i} \in \mathbb{R}^{(n-r) \times(n-r)}$ and $\Lambda_{i} \in \mathbb{R}^{(n-r) \times p}, i=1,2,3$ such that

$$
Q A_{i}=\Gamma_{i} Q+\Lambda_{i} C \quad i=1,2,3 .
$$


III. There exists $G=\left[G_{1} G_{2} G_{3}\right] \in \mathbb{R}^{n \times 3 p}$ such that

$$
\left(A_{H}+G C_{D}\right) \mathcal{V} \dot{\oplus} \mathcal{V} \dot{\oplus} \mathcal{V} \subseteq \mathcal{V}
$$

or equivalently there exist $G_{i} \in \mathbb{R}^{n \times p}$ such that

$$
\left(A_{i}+G_{i} C\right) \mathcal{V} \subseteq \mathcal{V} \quad \text { for } i=1,2,3
$$

Proof The proof follows from an argument similar to that used in Lemma 3.1 of $[16]$.

3.2 Input-containing $\left(A_{H}, C_{D}\right)$-conditioned invariants and their characterization

The framework for fault isolation constructed later on in the paper depends on the concept of input-containing subspaces will play an important role. The definition is as follows.

Definition $5 \mathcal{V} \subset \mathbb{R}^{n}$ is an input-containing conditioned invariant subspace for (1) if

$$
\left[A_{H} B_{H}\right]\left(\left(\mathcal{V} \dot{\oplus} \mathcal{V} \dot{\oplus} \mathcal{V} \dot{\oplus} \mathbb{R}^{3 m}\right) \cap \operatorname{ker}\left[\begin{array}{ll}
C_{D} & 0_{3 p \times 3 m}
\end{array}\right]\right) \subseteq \mathcal{V}
$$

The following characterizations of input-containing subspaces hold.

Proposition 4 Let $\mathcal{V}$ be a $r$-dimensional subspace of $\mathbb{R}^{n}$, and let $Q \in \mathbb{R}^{(n-r) \times n}$ be a full rank matrix such that $\operatorname{ker}(Q)=\mathcal{V}$. The following statements are equivalent:

I. The subspace $\mathcal{V}$ is an input-containing conditioned invariant for (1);

II. There exist $\Gamma=\left[\begin{array}{lll}\Gamma_{1} & \Gamma_{2} & \Gamma_{3}\end{array}\right] \in \mathbb{R}^{(n-r) \times 3(n-r)}$ and $\Lambda \in\left[\begin{array}{lll}\Lambda_{1} & \Lambda_{2} & \Lambda_{3}\end{array}\right] \in$ $\mathbb{R}^{(n-r) \times 3 p}$ such that

$$
Q\left[\begin{array}{ll}
A_{H} & B_{H}
\end{array}\right]=\Gamma\left[\begin{array}{ll}
Q_{D} & 0_{3(n-r) \times 3 m}
\end{array}\right]+\Lambda\left[\begin{array}{ll}
C_{D} & 0_{3 p \times 3 m}
\end{array}\right],
$$

or equivalently there exist $\Gamma_{i} \in \mathbb{R}^{(n-r) \times(n-r)}$ and $\Lambda_{i} \in \mathbb{R}^{(n-r) \times p}, i=1,2,3$ such that

$$
Q\left[A_{i} B_{i}\right]=\Gamma_{i}\left[\begin{array}{ll}
Q & 0_{(n-r) \times m}
\end{array}\right]+\Lambda_{i}\left[\begin{array}{ll}
C & 0_{p \times m}
\end{array}\right] \quad i=1,2,3 .
$$

III. There exists $G=\left[G_{1} G_{2} G_{3}\right] \in \mathbb{R}^{n \times 3 p}$ such that

$$
\left[A_{H}+G C_{D} B_{H}\right]\left(\mathcal{V} \dot{\oplus} \mathcal{V} \dot{\oplus} \mathcal{V} \dot{\oplus} \mathbb{R}^{3 m}\right) \subseteq \mathcal{V}
$$

or, equivalently, there exist $G_{i} \in \mathbb{R}^{n \times p}$ such that

$$
\left[A_{i}+G_{i} C B_{i}\right]\left(\mathcal{V} \dot{\oplus} \mathbb{R}^{m}\right) \subseteq \mathcal{V} \quad \text { for } i=1,2,3
$$


Proof $((I) \Longrightarrow(I I))$ : Condition $(I)$ is equivalent to

$$
\left[\begin{array}{ll}
A_{H} & B_{H}
\end{array}\right] \operatorname{ker}\left[\begin{array}{cc}
Q_{D} & 0_{3(n-r) \times 3 m} \\
C_{D} & 0_{3 p \times 3 m}
\end{array}\right] \subseteq \operatorname{ker} Q .
$$

The following lemma can be proved using standard linear algebra.

Lemma 1 Let $X: \mathbb{R}^{n} \rightarrow \mathbb{R}^{m}$ be a linear map, and let $\mathcal{V} \subseteq \mathbb{R}^{n}, \mathcal{V}^{\prime} \subseteq \mathbb{R}^{m}$ be subspaces of dimension $r$ and $r^{\prime}$, respectively. Let $Z \in \mathbb{R}^{(n-r) \times(n-r)}, Y \in$ $\mathbb{R}^{\left(m-r^{\prime}\right) \times\left(m-r^{\prime}\right)}$ be such that $\operatorname{ker}(Z)=\mathcal{V}$ and $\operatorname{ker}(Y)=\mathcal{V}^{\prime}$. Then

$$
X \mathcal{V} \subseteq \mathcal{V}^{\prime} \Longleftrightarrow \exists L \text { such that } Y X=L Z .
$$

As a consequence of Lemma 1 it follows $Q\left[\begin{array}{ll}A_{H} & B_{H}\end{array}\right]=L\left[\begin{array}{cc}Q_{D} & 0_{3(n-r) \times 3 m} \\ C_{D} & 0_{3 p \times 3 m}\end{array}\right]$ for some $L \in \mathbb{R}^{(n-r) \times 3(n-r)+3 p}$. Now partition $L$ conformably with

$$
\left[\begin{array}{cc}
Q_{D} & 0_{3(n-r) \times 3 m} \\
C_{D} & 0_{3 p \times 3 m}
\end{array}\right]
$$

as $L:=\left[\begin{array}{ll}\Gamma & \Lambda\end{array}\right]$ where $\Gamma=\left[\begin{array}{lll}\Gamma_{1} & \Gamma_{2} & \Gamma_{3}\end{array}\right] \in \mathbb{R}^{(n-r) \times 3(n-r)}$ and $\Lambda \in\left[\begin{array}{lll}\Lambda_{1} & \Lambda_{2} & \Lambda_{3}\end{array}\right] \in$ $\mathbb{R}^{(n-r) \times 3 p}$, then it is immediate to verify that (11) holds.

$$
\begin{aligned}
((I I) \Longrightarrow & (I I I)): \text { Let } x \in \mathcal{V} \dot{\oplus} \mathcal{V} \dot{\oplus} \mathcal{V} \dot{\oplus} \mathbb{R}^{3 m}, \text { then } \\
& Q\left[A_{H} B_{H}\right] x=\Gamma \underbrace{\left[\begin{array}{ll}
Q_{D} & 0_{3(n-r) \times 3 m}
\end{array}\right] x}_{=0}+\Lambda\left[\begin{array}{ll}
C_{D} & 0_{3(n-r) \times 3 m}
\end{array}\right] x .
\end{aligned}
$$

Consequently, $\left(Q\left[\begin{array}{ll}A_{H} & B_{H}\end{array}\right]-\Lambda\left[\begin{array}{ll}C_{D} & 0_{3(n-r) \times 3 m}\end{array}\right]\right) x=0$. Let $G^{\prime} \in \mathbb{R}^{n \times(n-r)}$ be a right-inverse of $Q$; it follows $Q\left(\left[A_{H} B_{H}\right]-G^{\prime} \Lambda\left[C_{D} 0_{2(n-r) \times 3 m}\right]\right) x=0$. Now define $G:=-G^{\prime} \Lambda$. Then

$$
Q\left(\left[A_{H}+G C_{D} B_{H}\right]\right) x=0
$$

which proves the claim.

$$
\begin{gathered}
((I I I) \Longrightarrow(I)): \text { Let } x \in \mathcal{V} \dot{\oplus} \mathcal{V} \dot{\oplus} \mathcal{V} \dot{\oplus} \mathbb{R}^{3 m} \bigcap \text { ker }\left[C_{D} 0_{3 p \times 3 m}\right] . \text { Then } \\
{\left[A_{H}+G C_{D} B_{H}\right] x=\left[\begin{array}{ll}
A_{H} & B_{H}
\end{array}\right] x \in \mathcal{V},}
\end{gathered}
$$

which proves (13). Hence (I) follows from Definition 5 .

The intersection of two input-containing subspaces is also input containing; thus the smallest input-containing subspace of $\left(A_{i}, B_{i}, C\right), i=1,2,3$ is the intersection of all input-containing subspaces of $\left(A_{i}, B_{i}, C\right), i=1,2,3$. Denote by $\mathcal{W}(\mathcal{B})^{\star}$ the smallest $\left(A_{H}, C_{D}\right)$-conditioned invariant containing $\operatorname{im}(B)=: \mathcal{B}$. To compute $\mathcal{W}(\mathcal{B})^{\star}$ the recursion (see Algorithm 4.1 p. 89 of [16]) is used: 


$$
\mathcal{W}_{i}:= \begin{cases}\{0\} & \text { if } i=0 \\
{\left[A_{H} B_{H}\right]\left(\mathcal{W}_{i-1} \dot{\oplus} \mathcal{W}_{i-1} \dot{\oplus} \mathcal{W}_{i-1} \dot{\oplus} \mathbb{R}^{3 m} \cap \operatorname{ker}\left[\begin{array}{ll}
C_{D} & 0_{3 p \times 3 m}
\end{array}\right)\right)} & i>0\end{cases}
$$

then $\mathcal{W}(\mathcal{B})^{\star}=\lim _{i \rightarrow \infty} \mathcal{W}_{i}=\mathcal{W}_{n}$

\subsection{Unobservability subspaces}

Unobservability subspaces were introduced in the 1D case in [12]; they provide maximal freedom when choosing the dynamics of an asymptotic observer, and consequently are useful also in the 1D fault isolation problem. We now introduce the analogous $3 \mathrm{D}$ concept, which will be used later on in the paper to state necessary and sufficient conditions for fault identification.

The following is the definition of non-observable subspace [15] ( p. 350).

Definition 6 The non-observable subspace of $\left(A_{H}, C_{D}\right)$ is the limiting subspace of the sequence $\left\{\mathcal{N}_{i}\right\}_{i=0, \ldots}$ defined by

$$
\mathcal{N}_{i}:= \begin{cases}\operatorname{ker} C & \text { if } i=0 \\ \bigcap_{j=1,2,3} A_{j}{ }^{-1} \mathcal{N}_{i-1} \cap \operatorname{ker} C & \text { if } i>0\end{cases}
$$

where $A_{j}^{-1}$ is the inverse image of $A_{j}$. It follows from the definition that the non-observable subspace is $\left(A_{1}, A_{2}, A_{3}\right)$-invariant in the sense of Def. 1 ; indeed, it is the largest $A_{H}$-invariant subspace contained in ker $C$. It follows $a$ fortiori that it is also an $\left(A_{H}, C_{D}\right)$-conditioned invariant. In the following we denote the non-observable subspace of $\left(A_{H}, C_{D}\right)$ by $\mathcal{N}\left(A_{H}, C_{D}\right)$, or simply $\mathcal{N}$ when it is clear which matrices $A_{H}, C_{D}$ it corresponds to.

We now introduce the definition of a $3 \mathrm{D}$ unobservability subspace.

Definition 7 A subspace $\mathcal{S} \subseteq \mathbb{R}^{n}$ is a $\left(A_{H}, C_{D}\right)$-unobservability subspace for (1), if there exist $H \in \mathbb{R}^{\bullet \times 3 p}$ and $G=\left[G_{1} G_{2} G_{3}\right] \in \mathbb{R}^{n \times 3 p}$ such that $\mathcal{S}$ is the non-observable subspace of $\left(A_{H}+G C_{D}, H C_{D}\right)$.

Proposition 5 Let $\mathcal{S}$ be a $\left(A_{H}, C_{D}\right)$-unobservability subspace. Then $\mathcal{S}$ is an $\left(A_{H}, C_{D}\right)$-conditioned invariant.

Proof Consider the non-observable subspace of $\left(A_{H}+G C_{D}, H C_{D}\right)$ which is also $\left(A_{H}+G C_{D}, H C_{D}\right)$-invariant. From the equivalence of statement $\mathrm{I}$ and statement $I I I$ of Prop. 3 , it is also an $\left(A_{H}, H C_{D}\right)$-conditioned invariant. Since for all $H$ the inclusion ker $H C_{D} \supseteq$ ker $C_{D}$ holds, it also follows that each unobservability subspace is also an $\left(A_{H}, C_{D}\right)$-conditioned invariant.

Now let $\mathcal{L} \subset \mathbb{R}^{n}$ be a subspace. The set of all unobservability subspaces containing $\mathcal{L}$ is closed under intersection (see also the discussion at the end of section 3.2 ), and consequently there exists a smallest unobservability subspace containing $\mathcal{L}$; we denote it by $\mathcal{S}(\mathcal{L})^{\star}$. 
4 Internally- and externally-stabilizing gains, and their construction

Statement $I I I$ of Prop. 3 and statement $I I I$ of Prop. 4 show that analogously to the $1 \mathrm{D}$ and $2 \mathrm{D}$ cases, also in the $3 \mathrm{D}$ case (input-containing) $\left(A_{H}, C_{D}\right)$ conditioned invariance implies that an output-feedback matrix $G$ can be found that makes the subspace $\left(A_{1}+G_{1} C, A_{2}+G_{2} C, A_{3}+G_{3} C\right)$-invariant in the sense of Def. 1. In the design of asymptotic observers for fault detection, it is important to ensure that internal/external stability is also guaranteed. The purpose of this section is to show that the results of [16], stating that internal and external stability can be achieved by applying independent gain matrices, hold also for the 3D case; and that constructive (albeit probably conservative) procedures can be stated yielding internally/externally stabilizing gain matrices. Since for our purposes only input-containing conditioned invariant subspaces are relevant, we limit our treatment to this case, the general case being completely analogous.

4.1 Independence of internal and external stability for input-containing subspaces

Let $\mathcal{V}$ be an input-containing conditioned invariant subspace. Statement $I I I$ of Prop. 4 together with statement $I V$ of Prop. 1 imply the existence of a matrix $\widetilde{\Gamma}=\left[\begin{array}{lll}\widetilde{\Gamma_{1}} & \widetilde{\Gamma_{2}} & \widetilde{\Gamma}_{3}\end{array}\right]$ such that

$$
Q\left(\left[\begin{array}{ll}
A_{H} & B_{H}
\end{array}\right]+G\left[\begin{array}{ll}
C_{D} & 0_{3 p \times 3 m}
\end{array}\right]\right)=\left[\begin{array}{lll}
\widetilde{\Gamma_{1}} & \widetilde{\Gamma_{2}} & \Gamma_{3}
\end{array}\right]\left[\begin{array}{ll}
Q_{D} & 0_{3(n-r) \times 3 m}
\end{array}\right]
$$

or, equivalently,

$$
Q\left[A_{H} B_{H}\right]=[\widetilde{\Gamma}-Q G]\left[\begin{array}{cc}
Q_{D} & 0_{3(n-r) \times 3 m} \\
C_{D} & 0_{3 p \times 3 m}
\end{array}\right] .
$$

Denote by $H$ any full row-rank matrix such that ker $H=\operatorname{Im}\left[\begin{array}{l}Q_{D} \\ C_{D}\end{array}\right]$; then $[\widetilde{\Gamma}-Q G]$ is a solution of (15) if and only if there exists $K$ such that

$$
[\widetilde{\Gamma}-Q G]=Q\left[A_{H} B_{H}\right]\left[\begin{array}{cc}
Q_{D} & 0_{3(n-r) \times 3 m} \\
C_{D} & 0_{3 p \times 3 m}
\end{array}\right]^{\dagger}+K H .
$$

From statement II of Prop. 4 it follows that

$$
\left[\begin{array}{ll}
\Gamma & \Lambda
\end{array}\right]=Q\left[\begin{array}{ll}
A_{H} & B_{H}
\end{array}\right]\left[\begin{array}{cc}
Q_{D} & 0_{3(n-r) \times 3 m} \\
C_{D} & 0_{3 p \times 3 m}
\end{array}\right]^{\dagger}+K^{\prime} H
$$

for some matrix $K^{\prime}$. Comparing this expression with (16) we conclude that $\left[\begin{array}{ll}\Gamma & \Lambda\end{array}\right]-[\widetilde{\Gamma}-Q G]=\left(K^{\prime}-K\right) H$. Now partition $H$ as $H=\left[H^{\prime} H^{\prime \prime}\right]$, with 
$H^{\prime} \in \mathbb{R}^{\bullet \times 3(n-r)}$ and $H^{\prime \prime} \in \mathbb{R}^{\bullet \times 3 p}$; then $\Lambda=-Q G+\left(K^{\prime}-K\right) H^{\prime \prime}$. From this it follows that

$$
G=-Q^{\dagger} \Lambda+Q^{\dagger} \underbrace{\left(K^{\prime}-K\right)}_{=: K^{\prime \prime}} H^{\prime \prime}+\Omega U,
$$

where $\Omega$ is a full column rank matrix such that $\mathcal{V}=\operatorname{ker} Q=\operatorname{Im} \Omega$ and $U$ is an arbitrary matrix of suitable dimension.

Let $S$ be a nonsingular $n \times n$ matrix whose first $r$ columns span $\mathcal{V}$. Since $\mathcal{V}$ is an input-containing $\left(A_{1}+G_{1} C, A_{2}+G_{2} C, A_{3}+G_{3} C\right)$-invariant subspace, applying Theorem 1 yields

$$
S\left[A_{i}+G_{i} C\right] S^{-1}=\left[\begin{array}{cc}
\Delta_{i}^{11}\left(K^{\prime \prime}, U\right) & \Delta_{i}^{12}\left(K^{\prime \prime}, U\right) \\
0 & \Delta_{i}^{22}\left(K^{\prime \prime}, U\right)
\end{array}\right],
$$

where $U$ and $K^{\prime \prime}$ are two degrees of freedom that can be used to assign the inner dynamics of $\mathcal{V}$ by modifying $\Delta_{i}^{11}\left(K^{\prime \prime}, U\right)$ and the external dynamics of $\mathcal{V}$ by modifying $\Delta_{i}^{22}\left(K^{\prime \prime}, U\right)$. The following result shows that these dynamics can be assigned independently.

Proposition 6 For all $i \in\{1,2,3\}$, the matrix $\Delta_{i}^{22}\left(K^{\prime \prime}, U\right)$ in (18) does not depend on $U$, and the matrix $\Delta_{i}^{11}\left(K^{\prime \prime}, U\right)$ does not depend on $K^{\prime \prime}$.

Proof Let $U_{1}$ and $U_{2}$ be arbitrary matrices, and subtract the matrices (18) corresponding to the gains $-Q^{\dagger} \Lambda+Q^{\dagger} K^{\prime \prime} H^{\prime \prime}+\Omega U_{1}$ and $-Q^{\dagger} \Lambda+Q^{\dagger} K^{\prime \prime} H^{\prime \prime}+$

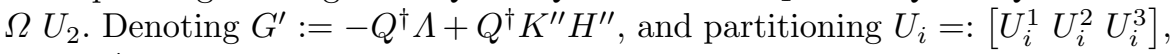
with $U_{i}^{j} \in \mathbb{R}^{r \times p}, i=1,2, j=1,2,3$, from (18) we obtain

$$
\begin{aligned}
& {\left[\begin{array}{cc}
\Delta_{i}^{11}\left(K^{\prime \prime}, U_{1}\right)-\Delta_{i}^{11}\left(K^{\prime \prime}, U_{2}\right) & \Delta_{i}^{12}\left(K^{\prime \prime}, U_{1}\right)-\Delta_{i}^{12}\left(K^{\prime \prime}, U_{2}\right) \\
0 & \Delta_{i}^{22}\left(K^{\prime \prime}, U_{1}\right)-\Delta_{i}^{22}\left(K^{\prime \prime}, U_{2}\right)
\end{array}\right] } \\
= & S\left[A_{i}+G_{i}^{\prime} C+\Omega U_{1}^{i} C\right] S^{-1}-S\left[A_{i}+G_{i}^{\prime} C+\Omega U_{2}^{i} C\right] S^{-1} \\
= & S \Omega\left[U_{1}^{i}-U_{2}^{i}\right] C S^{-1} .
\end{aligned}
$$

Without loss of generality, $S$ can be written as $S=\left[\begin{array}{l}V_{c} \\ Q\end{array}\right]$ for some suitable matrix $V_{c}$; consequently for (19)

$$
Q \Omega\left[U_{1}^{i}-U_{2}^{i}\right] C S^{-1}=\left[0 \Delta_{i}^{22}\left(K^{\prime \prime}, U_{1}\right)-\Delta_{i}^{22}\left(K^{\prime \prime}, U_{2}\right)\right],
$$

which by the definition of $\Omega$ equals to zero. Hence, $\Delta_{i}^{22}\left(K^{\prime \prime}, U_{1}\right)=\Delta_{i}^{22}\left(K^{\prime \prime}, U_{2}\right)$ which implies the term $\Delta_{i}^{22}\left(K^{\prime \prime}, U\right)$ in (18) does not depend on $U$.

To show that $\Delta_{i}^{11}\left(K^{\prime \prime}, U\right)$ does not depend on $K$, we proceed as follows. First, partition $H^{\prime \prime}=$ : $\left[\begin{array}{lll}H_{1}^{\prime \prime} & H_{2}^{\prime \prime} & H_{3}^{\prime \prime}\end{array}\right]$ with $H_{i}^{\prime \prime} \in \mathbb{R}^{\bullet \times p}, i=1,2,3$. Then use (18) and $S=\left[\begin{array}{l}V_{c} \\ Q\end{array}\right]$ to conclude that

$$
V_{c}\left[A_{i}-Q^{\dagger} \Lambda_{i} C+Q^{\dagger} K^{\prime \prime} H_{i}^{\prime \prime} C+\Omega U C\right]=\Delta_{i}^{11}\left(K^{\prime \prime}, U\right) V_{c}+\Delta_{i}^{12}\left(K^{\prime \prime}, U\right) Q .
$$


Now we compute (20) for $K^{\prime \prime}=K_{i}^{\prime \prime}, i=1,2$, and subtract the equations obtained in this way; after straightforward manipulations obtain

$$
\begin{aligned}
V_{c}\left[Q^{\dagger}\left(K_{1}^{\prime \prime}-K_{2}^{\prime \prime}\right) H_{i}^{\prime \prime} C\right] & =\left(\Delta_{i}^{11}\left(K_{1}^{\prime \prime}, U\right)-\Delta_{i}^{11}\left(K_{2}^{\prime \prime}, U\right)\right) V_{c} \\
& +\left(\Delta_{i}^{12}\left(K_{1}^{\prime \prime}, U\right)-\Delta_{i}^{12}\left(K_{2}^{\prime \prime}, U\right)\right) Q .
\end{aligned}
$$

From $H\left[\begin{array}{l}Q_{D} \\ C_{D}\end{array}\right]=H^{\prime} Q_{D}+H^{\prime \prime} C_{D}=0$ it follows that the subspace spanned by the rows of $H^{\prime \prime} C_{D}$ is a subspace of the row span of $Q$. Since $V_{c}$ and $Q$ have linearly independent rows, from (21) it follows that $\Delta_{i}^{11}\left(K_{1}^{\prime \prime}, U\right)-\Delta_{i}^{11}\left(K_{2}^{\prime \prime}, U\right)=$ 0 ; consequently, $\Delta_{i}^{11}(K, U)$ in (18) does not depend on $K$.

4.2 Construction of internally and externally stabilizing gains for input-containing subspaces

We now consider the problem of constructing internally- and externally stabilizing gains for a given input-containing $\left(A_{H}, C_{D}\right)$-invariant subspace. As the following result shows, external stability is equivalent to the existence of $\Gamma=\left[\begin{array}{lll}\Gamma_{1} & \Gamma_{2} & \Gamma_{3}\end{array}\right] \in \mathbb{R}^{(n-r) \times 3(n-r)}$ and $\Lambda=\left[\Lambda_{1} \Lambda_{2} \Lambda_{3}\right] \in \mathbb{R}^{(n-r) \times 3 p}$ such that $\Gamma_{i}$ has all its eigenvalues inside the open unit circle in the complex plane.

Proposition 7 Let $\Gamma$, $\Lambda$ satisfy (11). Then $\Gamma_{i}=\Delta_{i}^{22}\left(K^{\prime \prime}, U\right)$, the $(2,2)$-block of (18).

Proof From statement II of Prop. 4 we conclude that

$$
Q\left[A_{i} B_{i}\right]-\Lambda_{i}\left[C 0_{p \times m}\right]=\Gamma_{i}\left[Q 0_{(n-r) \times m}\right],
$$

$i=1,2,3$, from which we obtain

$$
Q(\left[A_{i} B_{i}\right] \underbrace{-\left(Q^{\dagger}+V K\right) \Lambda_{i}}_{=: G_{i}}\left[C 0_{p \times m}\right])=\Gamma_{i}\left[Q 0_{(n-r) \times m}\right],
$$

where $\operatorname{Im} V=\mathcal{V}$ and $K$ is an arbitrary matrix of suitable dimensions. Now consider (18), and partition $S$ as $S=\left[\begin{array}{l}V_{c} \\ Q\end{array}\right]$, as in the proof of Prop. 6. The second block row of (19) yields $Q\left[A_{i}+G_{i} C\right] S^{-1}=\left[0 \Delta_{i}^{22}\left(K^{\prime \prime}, U\right)\right]=\Gamma_{i} Q S^{-1}$. Conclude that $\Gamma_{i} Q=\left[0 \Delta_{i}^{22}\left(K^{\prime \prime}, U\right)\right] S=\Delta_{i}^{22}\left(K^{\prime \prime}, U\right) Q$. Since $Q$ has full rowrank, this implies $\Gamma_{i}=\Delta_{i}^{22}\left(K^{\prime \prime}, U\right)$.

The following is an immediate consequence of Prop. 7. 
Corollary 1 Let $\mathcal{V}$ be an input-containing $\left(A_{H}, C_{D}\right)$-conditioned invariant subspace of dimension $r$, and denote by $Q \in \mathbb{R}^{(n-r) \times n}$ a full row rank matrix such that $\operatorname{ker}(Q)=\mathcal{V} . \mathcal{V}$ is externally stabilizable if and only if there exists $G_{i} \in \mathbb{R}^{n \times p}, i=1,2,3$, such that $Q\left(A_{i}+G_{i} C\right)=\Gamma_{i} Q$ with $\Gamma_{i} \in \mathbb{R}^{(n-r) \times(n-r)}$ Schur in the sense of $[6]$ p.487, $i=1,2,3$.

The matrix $\Delta_{i}^{11}$ in (18) is related to the internal stability properties of the conditioned invariant subspace, as the following result shows.

Proposition 8 Let $\mathcal{Q}$ be the subspace spanned by the rows of $Q$ such that $\mathcal{Q} \oplus \mathcal{Q}^{\perp}=\mathbb{R}^{(n-r)}$ and let $V_{c} \in \mathbb{R}^{r \times n}$ be such that $\left[\begin{array}{l}V_{c} \\ Q\end{array}\right]$ is nonsingular and rows of $V_{c}$ form an orthonormal basis for $\mathcal{Q}^{\perp}$. Moreover $V_{c} V_{c}^{\top}=I_{r}$. Then $\Delta_{i}^{11}\left(K^{\prime \prime}, U_{i}\right)=V_{c}\left(A_{i}+\Omega U_{i} C\right) V_{c}^{\top}$.

Proof Multiply both sides of (18) on the right by $S V_{c}^{\top}$ to obtain

$$
S\left(A_{i}+G_{i} C\right) V_{c}^{\top}=\left[\begin{array}{cc}
\Delta_{i}^{11}\left(K^{\prime \prime}, U_{i}\right) & \Delta_{i}^{12}\left(K^{\prime \prime},{ }_{i}\right) \\
0 & \Delta_{i}^{22}\left(K^{\prime \prime}, U_{i}\right)
\end{array}\right] \underbrace{S V_{c}^{\top}}_{=\left[\begin{array}{l}
I \\
0
\end{array}\right]} .
$$

Hence, $S\left(A_{i}+G_{i} C\right) V_{c}^{\top}=\left[\Delta_{i}^{11}\left(K^{\prime \prime}, U\right) 0\right]^{\top}$, from which it follows that

$$
\Delta_{i}^{11}\left(K^{\prime \prime}, U\right)=V_{c}\left(A_{i}+G_{i} C\right) V_{c}^{T}
$$

From (17), and using the fact that the columns of $V_{c}^{\top}$ form an orthonormal basis for $\mathcal{V}$, it follows that $V_{c} G=V_{c} \Omega U$. The claim follows.

The following is an immediate consequence of Prop. 8.

Corollary 2 Let $\mathcal{V}$ be an $(A, C)$-conditioned invariant subspace of dimension $r$, and denote by $Q \in \mathbb{R}^{(n-r) \times n}$ a full row rank matrix such that $\operatorname{ker}(Q)=\mathcal{V}$. Moreover, let $V_{c} \in \mathbb{R}^{r \times n}$ be such that $\left[\begin{array}{l}V_{c} \\ Q\end{array}\right]$ is nonsingular, and $V_{c} V_{c}^{\top}=I_{r}$. $\mathcal{V}$ is internally stabilizable if and only if there exist $G_{i} \in \mathbb{R}^{n \times p}, i=1,2,3$, such that the triple

$$
\left(V_{c}\left(A_{1}+G_{1} C\right) V_{c}^{\top}, V_{c}\left(A_{2}+G_{2} C\right) V_{c}^{\top}, V_{c}\left(A_{3}+G_{3} C\right) V_{c}^{\top}\right)
$$

is stable.

To construct an externally stabilizing gain matrix $G$, we first compute, if exists, $\Gamma=\left[\begin{array}{lll}\Gamma_{1} & \Gamma_{2} & \Gamma_{3}\end{array}\right] \in \mathbb{R}^{(n-r) \times 3(n-r)}$ such that (11) holds for some $\Lambda \in$ $\mathbb{R}^{(n-r) \times 3 p}$, and moreover $\Gamma_{i}$ is Schur, $i=1,2,3$. Then we compute $G$ as a solution to $Q\left(A_{i}+G_{i} C\right)=\Gamma_{i} Q, i=1,2,3$. Note that from (11) it follows that

$$
\left[\begin{array}{ll}
\Gamma & \Lambda
\end{array}\right]=Q\left[\begin{array}{ll}
A_{H} & B_{H}
\end{array}\right]\left[\begin{array}{cc}
Q_{D} & 0_{3(n-r) \times 3 m} \\
C_{D} & 0_{3 p \times 3 m}
\end{array}\right]^{\dagger}+K H,
$$


where $H$ is any full row-rank matrix such that $\operatorname{ker}(H)=\operatorname{im}\left[\begin{array}{cc}Q_{D} & 0_{3(n-r) \times 3 m} \\ C_{D} & 0_{3 p \times 3 m}\end{array}\right]$. Denote

$$
\left[\begin{array}{llll}
V_{1} & V_{2} & V_{3} & \bar{V}
\end{array}\right]:=Q\left[\begin{array}{ll}
A_{H} & B_{H}
\end{array}\right]\left[\begin{array}{cc}
Q_{D} & 0_{3(n-r) \times 3 m} \\
C_{D} & 0_{3 p \times 3 m}
\end{array}\right]^{\dagger},
$$

where $V_{i} \in \mathbb{R}^{(n-r) \times(n-r)}, i=1,2,3$, and $\bar{V} \in \mathbb{R}^{(n-r) \times 3 p}$. Partition $H$ as

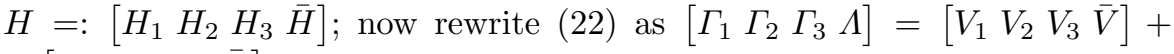
$K\left[H_{1} H_{2} H_{3} \bar{H}\right]$, from which it follows that $\Gamma_{i}=V_{i}+K H_{i}, i=1,2,3$, and $\Lambda=\bar{V}+K \bar{H}$.

Two cases are now possible, depending on whether $\left[\begin{array}{cc}Q_{D} & 0_{3(n-r) \times 3 m} \\ C_{D} & 0_{3 p \times 3 m}\end{array}\right]$ has full row-rank, or not.

In the first case $H=0$; consequently $\left[\begin{array}{ll}\Gamma & \Lambda\end{array}\right]=\left[\begin{array}{ll}A_{H} & B_{H}\end{array}\right]\left[\begin{array}{cc}Q_{D} & 0_{3(n-r) \times 3 m} \\ C_{D} & 0_{3 p \times 3 m}\end{array}\right]^{\dagger}$, with the matrix on the right-hand side being uniquely defined. This implies that if $\Gamma_{i}$ is Schur, $i=1,2,3$, then the corresponding $G$ makes $\mathcal{V}$ externally stable. Otherwise, no $G$ exists that makes $\mathcal{V}$ externally stable.

If $\left[\begin{array}{cc}Q_{D} & 0_{3(n-r) \times 3 m} \\ C_{D} & 0_{3 p \times 3 m}\end{array}\right]$ is not full row-rank, a matrix $K$ must be found such that $\Gamma_{i}=V_{i}+K H_{i}$ is asymptotically stable, $i=1,2,3$. In order to find such a $K$ we can use the result of Prop. 2 and try to solve the matrix inequality in the unknown positive-definite matrices $P_{i} \in \mathbb{R}^{n \times n}, i=1,2,3$ :

$$
\left[\begin{array}{ccc}
P_{1} & 0 & 0 \\
0 & P_{2} & 0 \\
0 & 0 & P_{3}
\end{array}\right]-\left[\begin{array}{c}
\Gamma_{1}^{\top} \\
\Gamma_{2}^{\top} \\
\Gamma_{3}^{\top}
\end{array}\right]\left(P_{1}+P_{2}+P_{3}\right)\left[\begin{array}{lll}
\Gamma_{1} & \Gamma_{2} & \Gamma_{3}
\end{array}\right]>0
$$

where $\Gamma_{i}=V_{i}+K H_{i}$. Note that (23) is not linear in $K$ and $P_{i}, i=1,2,3$; bilinear terms $P_{i} K$ appear. By introducing the auxiliary variables $\Phi_{1}:=P_{1}$, $\Phi_{2}:=P_{1}+P_{2}, \Phi_{3}:=P_{1}+P_{2}+P_{3}$, and using a Schur complement argument from (23) we conclude that

$$
\begin{aligned}
& {\left[\begin{array}{ccc}
\Phi_{1} & 0 & 0 \\
0 & \Phi_{2}-\Phi_{1} & 0 \\
0 & 0 & \Phi_{3}-\Phi_{2}
\end{array}\right]-\left[\begin{array}{c}
\Gamma_{1}^{\top} \\
\Gamma_{2}^{\top} \\
\Gamma_{3}^{\top}
\end{array}\right] \Phi_{3}\left[\begin{array}{lll}
\Gamma_{1} & \Gamma_{2} & \Gamma_{3}
\end{array}\right]>0} \\
& \Longleftrightarrow\left[\begin{array}{cccc}
\Phi_{1} & 0 & 0 & \Gamma_{1}^{\top} \Phi_{3} \\
0 & \Phi_{2}-\Phi_{1} & 0 & \Gamma_{2}^{\top} \Phi_{3} \\
0 & 0 & \Phi_{3}-\Phi_{2} & \Gamma_{3}^{\top} \Phi_{3} \\
\Phi_{3} \Gamma_{1} & \Phi_{3} \Gamma_{2} & \Phi_{3} \Gamma_{3} & \Phi_{3}
\end{array}\right]>0
\end{aligned}
$$

and introducing the auxiliary variable $\Theta:=\Phi_{3} K$, we conclude that (24) is equivalent with

$$
\begin{aligned}
& {\left[\begin{array}{cccc}
\Phi_{1} & 0 & 0 & \left(\Phi_{3} V_{1}+\Theta H_{1}\right)^{\top} \\
0 & \Phi_{2}-\Phi_{1} & 0 & \left(\Phi_{3} V_{2}+\Theta H_{2}\right)^{\top} \\
0 & 0 & \Phi_{3}-\Phi_{2} & \left(\Phi_{3} V_{3}+\Theta H_{3}\right)^{\top} \\
\Phi_{3} V_{1}+\Theta H_{1} & \Phi_{3} V_{2}+\Theta H_{2} & \Phi_{3} V_{3}+\Theta H_{3} & \Phi_{3}
\end{array}\right]>0} \\
& \Phi_{1}, \Phi_{2}, \Phi_{3}>0 .
\end{aligned}
$$


Having found solutions $\Psi_{i}, i=1,2,3$ and $\Theta$ to (25), the matrix $K$ is obtained as $K=\Phi_{3}^{-1} \Theta$.

To construct an internally stabilizing gain matrix $G$, we aim to compute the matrix $U$ with aid of the result of Prop. 2 i.e. we try to solve the matrix inequality in the unknown positive-definite matrices $P_{i} \in \mathbb{R}^{n \times n}, i=1,2,3$ :

$$
\left[\begin{array}{ccc}
P_{1} & 0 & 0 \\
0 & P_{2} & 0 \\
0 & 0 & P_{3}
\end{array}\right]-\left[\begin{array}{c}
Z_{1}^{\top} \\
Z_{2}^{\top} \\
Z_{3}^{\top}
\end{array}\right]\left(P_{1}+P_{2}+P_{3}\right)\left[\begin{array}{lll}
Z_{1} & Z_{2} & Z_{3}
\end{array}\right]>0
$$

where $Z_{i}:=V_{c}\left(A_{i}+\Omega U C\right) V_{c}^{T}$. Note that $(26)$ is not linear in $U$ and $P_{i}, i=$ $1,2,3$; by introducing auxiliary variables $\Psi_{1}:=P_{1}, \Psi_{2}:=P_{1}+P_{2}, \Psi_{3}:=P_{1}+$ $P_{2}+P_{3}$, and using a Schur's complement argument from (26) we conclude that

$$
\begin{aligned}
& {\left[\begin{array}{ccc}
\Psi_{1} & 0 & 0 \\
0 & \Psi_{2}-\Psi_{1} & 0 \\
0 & 0 & \Psi_{3}-\Psi_{2}
\end{array}\right]-\left[\begin{array}{c}
Z_{1}^{\top} \\
Z_{2}^{\top} \\
Z_{3}^{\top}
\end{array}\right] \Psi_{3}\left[Z_{1} Z_{2} Z_{3}\right]>0} \\
& \Longleftrightarrow\left[\begin{array}{cccc}
\Psi_{1} & 0 & 0 & Z_{1}^{\top} \Psi_{3} \\
0 & \Psi_{2}-\Psi_{1} & 0 & Z_{2}^{\top} \Psi_{3} \\
0 & 0 & \Psi_{3}-\Psi_{2} & Z_{3}^{\top} \Psi_{3} \\
\Psi_{3} Z_{1} & \Psi_{3} Z_{2} & \Psi_{3} Z_{3} & \Psi_{3}
\end{array}\right]>0
\end{aligned}
$$

and introducing the auxiliary variables $\Pi_{i}:=\Psi_{3} V_{c} \Omega U_{i}, i=1,2,3$ we conclude that $(28)$ is equivalent to

$$
\left[\begin{array}{ll}
T_{1} & T_{2}
\end{array}\right]>0
$$

where the matrices $T_{1}$ and $T_{2}$ are defined as:

$$
\begin{aligned}
T_{1}:= & {\left[\begin{array}{cc}
\Psi_{1} & 0 \\
0 & \Psi_{2}-\Psi_{1} \\
0 & 0 \\
\Psi_{3} V_{c} A_{1} V_{c}^{\top}+\Pi_{1} C V_{c}^{\top} & \Psi_{3} V_{c} A_{2} V_{c}^{\top}+\Pi_{2} C V_{c}^{\top}
\end{array}\right], } \\
0 & \left(\Psi_{3} V_{c} A_{1} V_{c}^{\top}+\Pi_{1} C V_{c}^{\top}\right)^{\top} \\
0 & \left(\Psi_{3} V_{c} A_{2} V_{c}^{\top}+\Pi_{2} C V_{c}^{\top}\right)^{\top} \\
T_{2}:= & {\left[\begin{array}{cc}
\Psi_{3}-\Psi_{2} & \left(\Psi_{3} V_{c} A_{3} V_{c}^{\top}+\Pi_{3} C V_{c}^{\top}\right)^{\top} \\
\Psi_{3} V_{c} A_{3} V_{c}^{\top}+\Pi_{3} C V_{c}^{\top} & \Psi_{3}
\end{array}\right] } \\
& \Psi_{1}, \Psi_{2}, \Psi_{3}>0 .
\end{aligned}
$$

Having found solutions $\Psi_{i}, i=1,2,3$ and $\Pi_{i}$ to (29), the matrix $U_{i}$ is given as $U_{i}=\Omega_{3}^{-1} V_{c}^{-1} \Psi_{3}^{-1} \Pi_{i}$ and finally $U=\left[U_{1} U_{2} U_{3}\right]$.

Example 1 Recently, FM state representations have been proposed for the modeling of wireless sensor networks (see [3]). The following set of matrices satisfies the conditions required for modeling such systems: 


$$
\begin{aligned}
& A_{1}=\left[\begin{array}{cccc}
-2 & \frac{-1}{4} & \frac{3}{2} & \frac{1}{4} \\
0 & \frac{-15}{4} & 0 & \frac{7}{4} \\
-3 & \frac{23}{4} & \frac{5}{2} & \frac{-11}{4} \\
0 & \frac{-7}{2} & 0 & \frac{3}{2}
\end{array}\right], A_{2}=\left[\begin{array}{cccc}
\frac{-16}{3} & \frac{55}{6} & \frac{17}{3} & \frac{-29}{6} \\
0 & \frac{-11}{6} & 0 & \frac{5}{6} \\
\frac{-34}{3} & \frac{139}{6} & \frac{35}{3} & \frac{-71}{6} \\
0 & \frac{-5}{3} & 0 & \frac{2}{3}
\end{array}\right], A_{3}=\left[\begin{array}{cccc}
\frac{-7}{5} & \frac{-217}{30} & \frac{6}{5} & \frac{109}{30} \\
0 & \frac{-59}{6} & 0 & \frac{29}{6} \\
\frac{-12}{5} & \frac{143}{30} & \frac{11}{5} & \frac{-71}{30} \\
0 & \frac{-29}{3} & 0 & \frac{14}{3}
\end{array}\right], \\
& B_{1}=B_{2}=0_{4 \times 2}, \quad B_{3}=\left[\begin{array}{ll}
3 & 4 \\
2 & 1 \\
1 & 7 \\
2 & 2
\end{array}\right], \quad C=\left[\begin{array}{rrcc}
1 & 0 & -1 & 0 \\
0 & -2 & 0 & 1 \\
0 & 0 & 1 & 0
\end{array}\right], \quad D=0_{3 \times 2} .
\end{aligned}
$$

We now compute a residual-generator that solves the asymptotic version of the problem.

Step 1. Using the recursion Algorithm (14) build $\mathcal{W}^{\star}$ :

$$
\mathcal{W}^{\star}=\left[\begin{array}{ll}
-0.7071 & -0.4781 \\
-0.4714 & -0.1195 \\
-0.2357 & -0.8367 \\
-0.4714 & -0.2390
\end{array}\right],
$$

such that the kernel of

$$
Q=\left[\begin{array}{cccc}
-0.7065 & 0.4837 & 0.1984 & 0.4770 \\
0.0000 & -0.6740 & -0.1123 & 0.7302
\end{array}\right]
$$

is exactly $\mathcal{W}^{\star}$.

Step 2. Construct an externally and internally stabilizing output injection matrix $G=\left[G_{1} G_{2} G_{3}\right]$ such that $\mathcal{W}^{\star}$ is an $\left(A_{i}+G_{i}\right)$-invariant input containing subspace, $i=1,2,3$ :

Since $\left[V_{1} V_{2} V_{3} \bar{V}\right]=Q\left[A_{H} B_{H}\right]\left[\begin{array}{cc}Q_{D} & 0 \\ C_{D} & D_{D}\end{array}\right]^{\dagger}$ and $\left[H_{1} H_{2} H_{3} \bar{H}\right]=\operatorname{im}\left[\begin{array}{ll}Q_{D} & 0 \\ C_{D} & D_{D}\end{array}\right]$, we obtain 


$$
\begin{aligned}
& V_{1}=\left[\begin{array}{ll}
-0.5067 & 0.3078 \\
-0.2010 & 0.0817
\end{array}\right], \quad V_{2}=\left[\begin{array}{ll}
-0.4432 & 0.8287 \\
-0.3871 & 0.6125
\end{array}\right], \quad V_{3}=\left[\begin{array}{ll}
-0.4397 & 0.4777 \\
-0.1709 & 0.1410
\end{array}\right], \\
& \bar{V}=\left[\begin{array}{ccccccccc}
0.4600 & 0.8567 & 0.0312 & 1.2070 & 1.3950 & -0.3015 & 0.2024 & 1.3879 & -0.0682 \\
0.1950 & 0.2608 & -0.0368 & 0.9996 & 0.9918 & -0.1654 & 0.1489 & 0.3942 & -0.0485
\end{array}\right], \\
& H_{1}=\left[\begin{array}{cc}
0 & -0.0000 \\
-0.4200 & 0.7678 \\
0 & 0.0000
\end{array}\right], H_{2}=\left[\begin{array}{cc}
-0.4069 & 0.7439 \\
-0.0000-0.0000 \\
-0.1040 & 0.1901
\end{array}\right], H_{3}=\left[\begin{array}{cc}
-0.1040 & 0.1901 \\
0 & 0 \\
0.4069 & -0.7439
\end{array}\right] \text {, } \\
& \bar{H}=\left[\begin{array}{ccccccccc}
-0.0000 & 0.0000 & 0.0000 & -0.2875 & -0.3491 & -0.1232 & -0.0735 & -0.0892 & -0.0315 \\
-0.2967 & -0.3603 & -0.1272 & -0.0000 & 0.0000 & -0.0000 & -0.0000 & -0.0000 & -0.0000 \\
0.0000 & -0.0000 & 0.0000 & -0.0735 & -0.0892 & -0.0315 & 0.2875 & 0.3491 & 0.1232
\end{array}\right] \text {. }
\end{aligned}
$$

Now by solving the LMI (25) for $\Phi_{i}, \Psi_{i}, K$ and $U_{i}, i=1,2,3$ we obtain

$$
\begin{aligned}
& \Phi_{1}=\left[\begin{array}{cc}
15.0115 & -0.14006 \\
-0.14006 & 15.2356
\end{array}\right], \Phi_{2}=\left[\begin{array}{cc}
22.0711 & -0.42019 \\
-0.42019 & 22.7433
\end{array}\right], \Phi_{3}=\left[\begin{array}{cc}
28.2383 & -1.1205 \\
-1.1205 & 30.0307
\end{array}\right], \\
& \Psi_{1}=\left[\begin{array}{cc}
16.91 & 0 \\
0 & 16.91
\end{array}\right], \quad \Psi_{2}=\left[\begin{array}{cc}
25.365 & 0 \\
0 & 25.365
\end{array}\right], \quad \Psi_{3}=\left[\begin{array}{cc}
33.82 & 0 \\
0 & 33.82
\end{array}\right] .
\end{aligned}
$$$$
K=\left[\begin{array}{lll}
-1.2186 & -0.5864 & 0.4317 \\
-0.8588 & -0.1921 & 0.0231
\end{array}\right]
$$$$
U=\left[\begin{array}{ccccccccc}
-0.1547 & 3.1568 & 0.6241 & -43.1943 & -49.0157 & -13.9290 & 73.3271 & 96.2555 & 32.1438 \\
6.8677 & 10.0520 & 2.3804 & 40.9437 & 49.2220 & 14.2804 & 1.1131 & 6.3128 & -0.0789
\end{array}\right] .
$$

Finally by exploiting the two degrees of freedom in constructing

$$
G=-Q^{\dagger}\left(Q Q^{\top}\right)^{-1} \Lambda+\Omega U
$$

, namely $K$ and $U$, we obtain an externally and internally stabilizing output injection matrix

$$
G=\left[G_{1} G_{2} G_{3}\right]
$$

where,

$$
\begin{aligned}
& G_{1}:=\left[\begin{array}{ccc}
-1.4980 & -4.2654 & -1.0348 \\
5.3591 & 5.8468 & 1.4753 \\
-2.5019 & -5.0023 & -1.0008
\end{array}\right], G_{2}:=\left[\begin{array}{ccc}
16.6137 & 18.0666 & 4.5725 \\
-4.8081 & -6.8264 & -2.0368 \\
2.6141 & 2.1981 & 1.1680
\end{array}\right] \text {, } \\
& G_{3}:=\left[\begin{array}{lll}
-47.1101 & -62.5076 & -20.5948 \\
-41.7793 & -51.2486 & -18.7387 \\
-28.9114 & -40.0829 & -12.3430
\end{array}\right]
\end{aligned}
$$




\section{Fault modeling for Fornasini-Marchesini models}

The nominal (i.e. fault-free) plant is assumed to be described by (1). To model the dynamics of the system after a sensor or actuator failure has occurred, following Massoumnia (see [12]) we augment the nominal model with additional terms that represent the failure modes:

$$
\begin{gathered}
\sigma_{1} \sigma_{2} \sigma_{3} x=A_{1} \sigma_{2} \sigma_{3} x+A_{2} \sigma_{1} \sigma_{3} x+A_{3} \sigma_{1} \sigma_{2} x \\
+B_{1} \sigma_{2} \sigma_{3} u+\left[L_{1}^{1} \ldots L_{1}^{k_{1}}\right] \sigma_{2} \sigma_{3}\left[\begin{array}{c}
m_{1}^{1} \\
\vdots \\
m_{1}^{k_{1}}
\end{array}\right] \\
+B_{2} \sigma_{1} \sigma_{3} u+\left[L_{2}^{1} \ldots L_{2}^{k_{2}}\right] \sigma_{1} \sigma_{3}\left[\begin{array}{c}
m_{2}^{1} \\
\vdots \\
m_{2}^{k_{2}}
\end{array}\right] \\
+B_{3} \sigma_{1} \sigma_{2} u+\left[L_{3}^{1} \ldots L_{3}^{k_{3}}\right] \sigma_{1} \sigma_{2}\left[\begin{array}{c}
m_{3}^{1} \\
\vdots \\
m_{3}^{k_{3}}
\end{array}\right] \\
y=C x+\left[J^{1} \ldots J^{p^{\prime}}\right]\left[\begin{array}{c}
n^{1} \\
\vdots \\
n^{p^{\prime}}
\end{array}\right],
\end{gathered}
$$

where $m_{i}^{k} \in\left(\mathbb{R}_{i}^{\ell_{i}^{k}}\right)^{\mathbb{Z}^{3}}, n^{j} \in\left(\mathbb{R}^{p_{j}}\right)^{\mathbb{Z}^{3}}$, and the matrices $L_{i}^{k} \in \mathbb{R}^{n \times \ell_{i}^{k}}, i=1,2,3$, $k=1, \ldots, k_{i}$ and $J^{k} \in \mathbb{R}^{p \times p_{k}}, k=1, \ldots, p^{\prime}$ are termed the actuator-and sensor failure signatures, respectively.

Under fault-free conditions $m_{i}^{k}=0$ and $n^{h}=0$ for $1 \leq i \leq 3,1 \leq k \leq$ $k_{i}$, and $1 \leq h \leq p^{\prime}$, and the model (30) reduces to (1). In order to model for example the effect of a complete failure in the $j$-th actuator in the $i$-th independent variable, set $L_{i}^{j}=B_{i}^{j}$ where $B_{i}^{j}$ is the $j$-th column of the input matrix $B_{i}$, and $m_{i}^{j}=-u_{i}^{j}$. Other types of failures (possibly affecting also the dynamics of the system as represented in the matrices $A_{i}, i=1,2,3$ ) can be accommodated in this framework; see section III of [12] for more details.

For simplicity of presentation in the rest of this paper we make the following assumptions:

Observability: The representation (1) is observable (in the sense of [4], p. $65)$;

Actuator-only faults: In (30) $J^{k}=0_{p \times p_{k}}, k=1, \ldots, p^{\prime}$;

Unambiguous failure modes: The failure signature matrix $L_{i}^{k}$ has full column rank, $1 \leq i \leq 3,1 \leq k \leq k_{i}$;

No simultaneous failures: If there exist $1 \leq \bar{i} \leq 3$ and $1 \leq \bar{k} \leq k_{i}$ such that $m_{\bar{i}}^{\bar{k}} \neq 0$, then $m_{i}^{h}=0$ for $i \neq \bar{i}$, and $h \neq \bar{k}$. 


\section{Failure detection and identification}

To perform failure detection and identification, we aim at designing an asymptotic observer for the nominal plant (1) and the failure model (30) that, under the assumptions stated at the end of Section 5, takes as inputs the input and output plant variables, and produces as output a residual which asymptotically provides information about the presence and the location of the failure. In this section we formalize this idea, and we provide necessary and sufficient conditions for the problem of fault identification stated in this way to be solvable.

The dynamics of the observer we will be designing are

$$
\begin{aligned}
\sigma_{1} \sigma_{2} \sigma_{3} \hat{x}= & A_{1} \sigma_{2} \sigma_{3} \hat{x}+A_{2} \sigma_{1} \sigma_{3} \hat{x}+A_{3} \sigma_{1} \sigma_{2} \hat{x}+B_{1} \sigma_{2} \sigma_{3} u+B_{2} \sigma_{1} \sigma_{3} u+B_{3} \sigma_{1} \sigma_{2} u \\
& -G_{1}\left(\sigma_{2} \sigma_{3} y-\sigma_{2} \sigma_{3} \hat{y}\right)-G_{2}\left(\sigma_{1} \sigma_{3} y-\sigma_{1} \sigma_{3} \hat{y}\right)-G_{3}\left(\sigma_{1} \sigma_{2} y-\sigma_{1} \sigma_{2} \hat{y}\right) \\
\hat{y}= & C \hat{x}
\end{aligned}
$$

where $G_{i} \in \mathbb{R}^{n \times p}, i=1,2,3$, are the gain matrices; $\hat{x}$ is the state estimate; and $\hat{y}=C \hat{x}$ is the output corresponding to it. We call the difference $\hat{x}-x$ the error vector, denoted by $e$, and we call $\hat{y}-y$ the residual vector, denoted by $r=C e$.

If no faults have occurred, the plant dynamics are described by (1), and consequently the error- and the residual dynamics are described by

$$
\begin{aligned}
\sigma_{1} \sigma_{2} \sigma_{3} e & =\left(A_{1}+G_{1} C\right) \sigma_{2} \sigma_{3} e+\left(A_{2}+G_{2} C\right) \sigma_{1} \sigma_{3} e+\left(A_{3}+G_{3} C\right) \sigma_{1} \sigma_{2} e \\
r & =C e .
\end{aligned}
$$

In the presence of an actuator fault described as in (30), subtracting (31) from (30) and rearranging the equations yields the following description of the error dynamics:

$$
\begin{aligned}
\sigma_{1} \sigma_{2} \sigma_{3} e= & \left(A_{1}+G_{1} C\right) \sigma_{2} \sigma_{3} e+\left(A_{2}+G_{2} C\right) \sigma_{1} \sigma_{3} e+\left(A_{3}+G_{3} C\right) \sigma_{1} \sigma_{2} e \\
& -\sum_{k=1}^{k_{1}} L_{1}^{k} \sigma_{2} \sigma_{3} m_{1}^{k}-\sum_{k=1}^{k_{2}} L_{2}^{k} \sigma_{1} \sigma_{3} m_{2}^{k}-\sum_{k=1}^{k_{3}} L_{3}^{k} \sigma_{1} \sigma_{2} m_{3}^{k} .
\end{aligned}
$$

Under the assumptions stated at the end of section 5, the error dynamics corresponding to a single failure in the $k$-th actuator of $B_{i}$ is described by

$$
\begin{aligned}
\sigma_{1} \sigma_{2} \sigma_{3} e= & \left(A_{1}+G_{1} C\right) \sigma_{2} \sigma_{3} e+\left(A_{2}+G_{2} C\right) \sigma_{1} \sigma_{3} e+\left(A_{3}+G_{3} C\right) \sigma_{1} \sigma_{2} e \\
& -L_{i}^{k} \sigma_{p} \sigma_{q} m_{i}^{k}, \quad i, p, q=1,2,3, \quad i \neq p \neq q .
\end{aligned}
$$

Disregarding for the moment the essential property of asymptotic stability for the observer (31), we now study the dynamics of (34) under zero boundary conditions $\left\{e\left(n_{1}, n_{2}, n_{3}\right)=0 \mid n_{1}+n_{2}+n_{3}=0\right\}$. 
6.1 A special case: zero boundary conditions

Let $G_{i} \in \mathbb{R}^{n \times p}, i=1,2,3$; for $\ell \in \mathbb{N}$, define the $\left(G_{1}, G_{2}, G_{3}\right)$-dependent matrices

$$
P_{i}^{k}(\ell):= \begin{cases}{\left[L_{i}^{k}\right]} & \ell=1 \\ {\left[\left(A_{i_{1}}+G_{i_{1}} C\right)\left(A_{i_{2}}+G_{i_{2}} C\right) \cdots\left(A_{i_{\ell}}+G_{i_{\ell}} C\right) L_{i}^{k}\right]} & \\ \left(i_{1}, \ldots, i_{\ell}\right) \in\{1,2,3\}_{\ell} & \ell>1\end{cases}
$$

where we assume the multi-indices $\left(i_{1}, \ldots, i_{\ell}\right)$ to be ordered e.g. lexicographically. It is a matter of straightforward verification to check that under the dynamics (34) with zero boundary conditions, the error vector $e\left(n_{1}, n_{2}, n_{3}\right)$ for $n_{1}+n_{2}+n_{3}=\ell$ belongs to $\operatorname{im} P_{i}^{k}(\ell), \ell=1, \ldots$ Define also the $\left(G_{1}, G_{2}, G_{3}\right)$ dependent matrix

$$
R_{i}^{k}:=\left[P_{i}^{k}(1) P_{i}^{k}(2) \ldots P_{i}^{k}(\ell) \ldots\right],
$$

and the associated $\left(G_{1}, G_{2}, G_{3}\right)$-dependent subspace

$$
\mathcal{V}_{i}^{k}:=\operatorname{im}\left(R_{i}^{k}\right) \text {. }
$$

The following is a geometric characterization of $\mathcal{V}_{i}^{k}$.

Proposition 9 Let $\mathcal{V}_{i}^{k}$ be defined as in (37), where $R_{i}^{k}$ is defined as in (36). Then $\mathcal{V}_{i}^{k \star}$ is the smallest $\left(A_{1}+G_{1} C, A_{2}+G_{2} C, A_{3}+G_{3} C\right)$-invariant subspace containing $\mathrm{im}\left(L_{i}^{k}\right)$.

Proof It follows from the definition of $P_{i}^{k}(\ell)$ that for $\ell \geq 1$ it holds that

$\operatorname{im} P_{i}^{k}(\ell+1)=\operatorname{im}\left[\left(A_{1}+G_{1} C\right) P_{i}^{k}(\ell)\left(A_{2}+G_{2} C\right) P_{i}^{k}(\ell)\left(A_{3}+G_{3} C\right) P_{i}^{k}(\ell)\right]$,

which implies the claim.

$\mathcal{V}_{i}^{k}$ is an input-containing $\left(A_{H}, C_{D}\right)$-conditioned invariant for the system described by

$$
\sigma_{1} \sigma_{2} \sigma_{3} x=A_{1} \sigma_{2} \sigma_{3} x+A_{2} \sigma_{1} \sigma_{3} x+A_{3} \sigma_{1} \sigma_{2} x-L_{i}^{k} \sigma_{2} \sigma_{3} m_{i}^{k},
$$

containing $\operatorname{im} L_{i}^{k}$.

We can now state our problem in the geometric language developed in the previous pages: find $G_{i} \in \mathbb{R}^{n \times p}, i=1,2,3$ such that the family $\mathcal{V}_{i}^{k}$ defined by (37) satisfies

$$
C \mathcal{V}_{\bar{i}}^{\bar{k}} \bigcap\left(\sum_{k \neq \bar{k}} C \mathcal{V}_{\bar{i}}^{k}+\sum_{i \neq \bar{i}} \sum_{k} C \mathcal{V}_{i}^{k}\right)=\{0\}
$$

for all $\bar{i}=1,2,3, \bar{k}=1, \ldots, q$. Note that $\mathcal{V}_{i}^{k}$ is $\left(A_{H}, C_{D}\right)$-invariant in the sense of Def. 4; then we can reformulate our problem equivalently as follows: 


\section{Residual generation problem with zero boundary conditions}

Find subspaces $\mathcal{V}_{i}^{k}, i=1,2,3, k=1, \ldots, k_{i}$, such that

(a) There exist $G_{i} \in \mathbb{R}^{n \times p}, i=1,2,3$ such that

$$
\left(A_{i}+G_{i} C\right) \mathcal{V}_{i}^{k} \subset \mathcal{V}_{i}^{k} \quad i=1,2,3, \quad k=1, \ldots, k_{i} ;
$$

(b) $\operatorname{im}\left(L_{i}^{k}\right) \subseteq \mathcal{V}_{i}^{k}$;

(c) $C \mathcal{V}_{\bar{i}}^{\bar{k}} \cap\left(\sum_{k \neq \bar{k}} C \mathcal{V}_{\bar{i}}^{k}+\sum_{i \neq \bar{i}} \sum_{k} C \mathcal{V}_{i}^{k}\right)=\{0\}$,

for all $\bar{i}=1,2,3, \bar{k}=1, \ldots, k_{i}$.

Now define $\mathcal{W}_{i}^{k \star}$ to be the smallest $\left(A_{H}, C_{D}\right)$-invariant subspace containing $\operatorname{im}\left(L_{i}^{k}\right)$; note that $\mathcal{W}_{i}^{k \star}$ only depends on $A_{i}, i=1,2,3, C$, and $\operatorname{im}\left(L_{i}^{k}\right) . \mathcal{W}_{i}^{k \star}$ can be computed in a manner analogous to the recursion (14). Recall also from section 3.3 the definition of $\mathcal{S}_{i}^{k \star}:=\mathcal{S}\left(\operatorname{im}\left(L_{i}^{k}\right)\right)^{\star}$, the smallest unobservability subspace containing $\operatorname{im}\left(L_{i}^{k}\right)$. The following result shows that our problem is solvable if and only if the family $\left\{\mathcal{W}_{i}^{k \star}\right\}$, or equivalently the family $\left\{\mathcal{S}_{i}^{k \star}\right\}$, satisfies condition $(c)$.

Theorem 2 The following statements are equivalent:

I. The residual generation problem with zero boundary conditions is solvable;

II. The family $\left\{\mathcal{W}_{i}^{k \star}\right\}_{\substack{i=1,2,3 ; \\ k=1, \ldots, k_{i}}}$ of smallest $\left(A_{H}, C_{D}\right)$-invariant subspaces containing $\operatorname{im}\left(L_{i}^{k}\right)$ satisfies condition $(c)$;

Proof $((I) \Longrightarrow(I I))$ Follows from the minimality of the $\mathcal{W}_{i}^{k \star}$, that satisfy $\mathcal{W}_{i}^{k \star} \subseteq \mathcal{V}_{i}^{k}, i=1,2,3, k=1, \ldots, q$ for any family $\left\{\mathcal{V}_{i}^{k}\right\}$ satisfying $(a)-(c)$.

$((I I) \Longrightarrow(I))$ By definition the $\mathcal{W}_{i}^{k \star}$ are $\left(A_{H}, C_{D}\right)$-invariant, but we need to prove that the same $G_{i} \in \mathbb{R}^{n \times p}, i=1,2,3$, can be found so that conditions $(a)-(c)$ are satisfied for $\mathcal{W}_{i}^{k \star}$. To this purpose, write each $\mathcal{W}_{i}^{k \star}$ as the following direct sum:

$$
\mathcal{W}_{i}^{k \star}=\mathcal{V}_{i}^{k \star} \dot{\oplus}\left(\mathcal{W}_{i}^{k \star} \bigcap \operatorname{ker} C\right),
$$

where $\mathcal{V}_{i}^{k \star}$ is some suitable subspace of $\mathbb{R}^{n}$, and let $W_{i}^{k \star} \in \mathbb{R}^{n \times \bullet}$ be a basis matrix for $\mathcal{W}_{i}^{k \star}$ structured according to such decomposition, i.e. $W_{i}^{k \star}=$ $\left[V_{i}^{k \star} W_{i}^{k \star \prime}\right]$, with the columns of $V_{i}^{k \star}$ spanning $\mathcal{V}_{i}^{k \star}$ and those of $W_{i}^{k \star \prime}$ spanning $\mathcal{W}_{i}^{k \star} \cap$ ker $C$. Observe that condition $(c)$ is equivalent to

$$
C \mathcal{V}_{\bar{i}}^{\bar{k} \star} \bigcap\left(\sum_{k \neq \bar{k}} C \mathcal{V}_{\bar{i}}^{k \star}+\sum_{i \neq \bar{i}} \sum_{k} C \mathcal{V}_{i}^{k \star}\right)=\{0\},
$$

for all $\bar{i}=1,2,3, \bar{k}=1, \ldots, k_{\bar{i}}$. This is equivalent to the matrix

$$
C\left[V_{1}^{1} \ldots V_{1}^{k_{1}} V_{2}^{1} \ldots V_{2}^{k_{2}} V_{3}^{1} \ldots V_{3}^{k_{3}}\right]
$$


having full column rank. Then each of the equations

$$
A_{i}\left[V_{1}^{1} \ldots V_{1}^{k_{1}} V_{2}^{1} \ldots V_{2}^{k_{2}} V_{3}^{1} \ldots V_{3}^{k_{3}}\right]=-G_{i} C\left[V_{1}^{1} \ldots V_{1}^{k_{1}} V_{2}^{1} \ldots V_{2}^{k_{2}} V_{3}^{1} \ldots V_{3}^{k_{3}}\right],
$$

$i=1,2,3$, has a solution $G_{i}$.

This concludes the proof of the Theorem.

Remark 1 With reference to the proof of Theorem 2, note that a set of gains $G_{i}, i=1,2,3$ can be computed by $G_{i}:=-A_{i} V\left[(C V)^{\top} C V\right]^{-1}(C V)^{\top}$, where $V:=\left[V_{1}^{1} \ldots V_{1}^{k_{1}} V_{2}^{1} \ldots V_{2}^{k_{2}} V_{3}^{1} \ldots V_{3}^{k_{3}}\right]$, and the columns of $\left[V_{i}^{1} \ldots V_{i}^{k_{i}}\right]$ form a basis for $\mathcal{W}_{i}^{k \star} \backslash\left(\mathcal{W}_{i}^{k \star} \cap\right.$ ker $\left.C\right)$. A similar procedure yields a set of gains for the family $\left\{\mathcal{S}_{i}^{k \star}\right\}$.

Remark 2 Given the current state of the art, it is unclear whether given a subspace $\mathcal{L}$, stabilizing $\mathcal{S}(\mathcal{L})^{\star}$ rather than $\mathcal{W}(\mathcal{L})^{\star}$ gives any more freedom in the assignment of the external dynamics of an asymptotic observer, as it is the case with 1D observers. However, when we consider the former choice in the light of Theorem 2, we need to solve an LMI (see section 4.2) of smaller dimension.

\subsection{The general case: asymptotic observers for fault detection}

The result of Theorem 2 is a necessary structural requirement on the system: unless the subspaces $\mathcal{W}_{i}^{k \star}$ (equivalently, $\mathcal{S}_{i}^{k \star}$ ) satisfy condition $(c)$, even in the special case of zero error on the boundary conditions of the system, i.e. $x\left(n_{1}, n_{2}, n_{3}\right)=\hat{x}\left(n_{1}, n_{2}, n_{3}\right)$ for all $\left(n_{1}, n_{2}, n_{3}\right)$ such that $n_{1}+n_{2}+n_{3}=0$, fault detection with an observer (31) is impossible. The case in which the boundary conditions of the observer are exactly the same as those of the plant hardly ever occurs, and we need to introduce stability in our problem formulation.

It is easy to verify that the sum of conditioned invariants is in general not a conditioned invariant. However, the following result shows that this property holds for the subspaces $\mathcal{W}_{i}^{k \star}$ defined in Theorem 2 (see also Lemma 4 p. 842 of [12]).

Proposition 10 Let $\mathcal{W}_{i}^{k \star}, i=1,2,3, k=1, \ldots, k_{i}$, be the smallest $\left(A_{H}, C_{D}\right)$ invariant subspace containing $\operatorname{im}\left(L_{i}^{k}\right)$. Denote by $\mathcal{W}^{\star}$ the smallest $\left(A_{H}, C_{D}\right)$ invariant subspace containing $\sum_{i=1}^{3} \sum_{k=1}^{k_{i}} \operatorname{im}\left(L_{i}^{k}\right)$. Assume that the family $\left\{\mathcal{W}_{i}^{k \star}\right\}$ satisfies condition (c); then

$$
\mathcal{W}^{\star}=\sum_{i=1}^{3} \sum_{k=1}^{k_{i}} \mathcal{W}_{i}^{k \star}
$$

Proof We first show that $\mathcal{W}^{\star} \subseteq \sum_{i=1}^{3} \sum_{k=1}^{k_{i}} \mathcal{W}_{i}^{k \star}$. Use the argument of the implication $(I I) \Longrightarrow(I)$ in Theorem 2 to prove that there exist $G_{i}$ such that $\mathcal{W}_{i}^{k \star}$ is $\left(A_{i}+G_{i} C\right)$-invariant, $i=1,2,3$. This implies that $\sum_{i=1}^{3} \sum_{k=1}^{k_{i}} \mathcal{W}_{i}^{k \star}$ is also 
an $\left(A_{H}, C_{D}\right)$-invariant. Of course this subspace contains $\sum_{i=1}^{3} \sum_{k=1}^{k_{i}} \operatorname{im}\left(L_{i}^{k}\right)$, and since $\mathcal{W}^{\star}$ is the smallest $\left(A_{H}, C_{D}\right)$-invariant containing it, the inclusion follows.

In order to prove the converse inclusion, observe that for all $\bar{i}=1,2,3$ and $\bar{k}=1, \ldots, k_{i}$, it holds that $\operatorname{im}\left(L_{\bar{i}}^{\bar{k}}\right) \subset \sum_{i=1}^{3} \sum_{k=1}^{k_{i}} \operatorname{im}\left(L_{i}^{k}\right)$. Since $\mathcal{W}^{\star}$ is $\left(A_{H}, C_{D}\right)$-invariant, it follows then that $\mathcal{W}_{\bar{i}}^{\bar{k} \star} \subset \mathcal{W}^{\star}$, and consequently the required inclusion also holds. This concludes the proof.

We say that an observer (31) solves the asymptotic residual generation problem if for arbitrary boundary conditions $\hat{x}_{\mid \mathcal{N}_{k}}$, asymptotically the residual $r$ is either zero (if there is no fault) or (if a fault occurs) it belongs to one, and only one, of the subspaces $C \mathcal{W}_{i}^{k \star}$, thus allowing the unique identification of the fault. A sufficient condition is spelled out in the following result.

Theorem 3 Let $\mathcal{W}_{i}^{k \star}, i=1,2,3, k=1, \ldots, k_{i}$, be the smallest $\left(A_{H}, C_{D}\right)$ invariant subspace containing $\operatorname{im}\left(L_{i}^{k}\right)$. Denote by $\mathcal{W}^{\star}$ the smallest $\left(A_{H}, C_{D}\right)$ invariant subspace containing $\sum_{i=1}^{3} \sum_{k=1}^{k_{i}} \operatorname{im}\left(L_{i}^{k}\right)$. Assume the family $\left\{\mathcal{W}_{i}^{k \star}\right\}$ satisfies condition $(c)$, and that $\mathcal{W}^{\star}$ is internally and externally stabilizable. Then there exist $G_{i} \in \mathbb{R}^{n \times p}, i=1,2,3$, such that the observer (31) solves the asymptotic residual generation problem.

Proof Since $\mathcal{W}^{\star}$ is an internally and externally $\left(A_{H}, C_{D}\right)$-conditioned invariant, we can find gain matrices $G_{i} \in \mathbb{R}^{n \times p}, i=1,2,3$, to construct an observer (31). We now consider how the observer functions in the two situations when there is no fault, or when a fault has occurred.

Assume that no fault has occurred; then the dynamics of the error are described by $(32)$. Since $\mathcal{W}^{\star}$ is externally stable, the dynamics of the error due to the component of the boundary conditions $\hat{x}_{\mid \mathcal{N}_{0}}$ lying outside of $\mathcal{W}^{\star}$ is asymptotically stable, and consequently goes to zero. Since $\mathcal{W}^{\star}$ is internally stable, the dynamics of the error due to the component of the boundary conditions in $\mathcal{W}^{\star}$ is also asymptotically stable, and consequently goes to zero. Conclude that asymptotically the error vector is zero, and consequently that the residual is also zero.

Now consider instead the case when one fault has occurred, for example corresponding to the error signature $L_{i}^{k}$; then the dynamics of the error is described by (34), with $m_{i}^{k} \in\left(\mathbb{R}^{\ell_{i}^{k}}\right)^{\mathbb{Z}^{3}}$ nonzero. Asymptotically the error vector lies in $\mathcal{W}^{\star}$; consequently the residual corresponding to it lies in the subspace $C \mathcal{W}^{\star}=C\left(\sum_{i=1}^{3} \sum_{k=1}^{k_{i}} \mathcal{W}_{i}^{k \star}\right)$. Since condition $(c)$ holds, it is possible by projecting the residual onto the subspaces $C \mathcal{W}_{i}^{k \star}$ to determine which type of fault the residual corresponds to.

\section{Conclusions and further work}

Following [12], we have outlined a framework for fault detection in 3D systems based on the geometric concepts generalized to the 2D case in [14-16] and 
some generalizations thereof. The basic results of this paper are Th. 2, which gives structural necessary conditions for fault detection to be possible, and Th. 3, where sufficient conditions are given for the existence of an observer to perform fault detection. The analysis in this paper considers the 3D case as the longer term research goal is to employ multidimensional systems theory in the analysis and design of grid sensor networks, which can be viewed as 3D systems. The results given generalize in a natural manner to $n \mathrm{D}$ with $n>3$ and hence the details have been omitted in the interests of brevity.

The results in this paper use the geometric approach to address the existence question and require further development in a number of areas. One of these is to find necessary and sufficient conditions, if they exist, as opposed to sufficient for key results, such as Th. 3) for the solvability of the problem, which in turn rely only on sufficient conditions (see Prop. 2) for the existence of stabilizing gains. The issue of how conservative our conditions are, and consequently how robust with respect to modeling errors and disturbances a fault detection scheme based on the principles used in this paper is, of course, directly linked to this problem. The results have been developed in the D systems setting with grid sensor networks as the target application but extension to the $n \mathrm{D}$ case, $n>3$, is straightforward.

These are important issues that need to be addressed to make this approach realistic for application to real-life situations. Another aspect to consider is that the approach used in this paper still treats all independent variables to be on an equal footing, while for most applications one of the independent variable is time. A "time-relevant" fault estimation framework is needed, where the distinguished role of the independent variable time in the modeling of the fault and in its estimation is recognized and exploited to provide better performance (on time-relevant systems, see also $[13,17]$ ).

\section{References}

1. Basile, G. and G. Marro, Controlled and Conditioned Invariants in Linear System Theory, Prentice-Hall, 1992.

2. Bisiacco, M. and M.E. Valcher, "Observer-based fault detection and isolation for 2D state-space models", Multidim. Syst. Sign. Proc., vol. 17, pp. 219-242, 2006.

3. Buddika Sumanasena, M.G. and P.H. Bauer, "Realization Using the Fornasini-Marchesini Model for Implementations in Distributed Grid Sensor Networks", IEEE Trans. Circ. Syst.- I, vol. 58, no. 11, 2011.

4. Fornasini, E., Marchesini, G., "Doubly-indexed dynamical systems: State-space models and structural properties", Mathematical Systems Theory, pp. 59-72, vol. 12, no. 1, 1978.

5. Fornasini, E., Marchesini, G. "Failure detection in 2D systems", Control-Theory and Advanced technology, vol. 4, no. 4, 381-393, 1988.

6. Graham, R.L. and Lehmer, D.H. "On the permanent of Schur's matrix", Journal of the Australian mathematical society, soc. 21, pp.487-497, 1975

7. Gałkowski, K., Lam, J., Xu, S. and Z. Lin, "LMI approach to state-feedback stabilization of multidimensional systems", Int. J. Contr., vol. 76, no. 14, pp. 1428-1436, 2003.

8. Hwang I., Kim, S., Kim, Y., and Seah, C.E. "A Survey of Fault Detection, Isolation, and Reconfiguration Methods", IEEE Trans. Contr. Syst. Techn., vol. 18, no. 3, pp. 636-653, 2010 . 
9. Ihab, S., Postlethwaite, I. and Gu, Dai-Wei, "Survey and application of sensor fault detection and isolation schemes", Control Engineering Practice, vol. 19, no. 7, pp. 658$674,2011$.

10. Jury, E.I., "Stability of multidimensional scalar and matrix polynomials", Proc. IEEE, vol.66, no.9, pp. 1018-1047, 1978.

11. Kar, H. and V. Singh, "Stability of 2-D systems described by the Fornasini-Marchesini first model", IEEE Trans. Sign. Proc., vol. 51, no.6, pp. 1675-1676, 2003.

12. M.A. Massoumnia, "A Geometric Approach to the Synthesis of Failure Detection Filters", IEEE Trans. Aut. Contr., pp.839-849, vol. AC-31, 1986.

13. Napp-Avelli, D. Rapisarda, P., and Rocha, P., "Time-relevant stability of 2D systems", Automatica, vol. 47, no. 11, pp. 2373-2382, 2011.

14. Ntogramatzidis, L., Cantoni, M. and Ran Y., "A geometric theory for 2-D systems including notions of stabilizability", Multidim. Syst. Signal Process., pp.449-475, vol. 19, 2008.

15. Ntogramatzidis, L., "Structural invariants of two-dimensional systems", SIAM J. Contr. Opt., vol. 50, no. 1, pp. 334-356, 2012.

16. Ntogramatzidis, L. and Cantoni, M., "Detectability subspaces and observer synthesis for two-dimensional systems", Multidim. Syst. Signal Process., pp.79-96, vol. 23, 2012.

17. Rapisarda, P. and Rocha, P. "Lyapunov functions for time-relevant 2D systems, with application to first-orthant stable systems", Automatica, vol. 48, no. 9, pp. 1998-2006, 2012.

18. W.M. Wonham, Linear multivariable control: a geometric approach, Springer-Verlag, 1979.

19. Zampieri, S., "2D residual generation and dead beat observers", Syst. Contr. Lett., vol. $17,483492,1992$. 